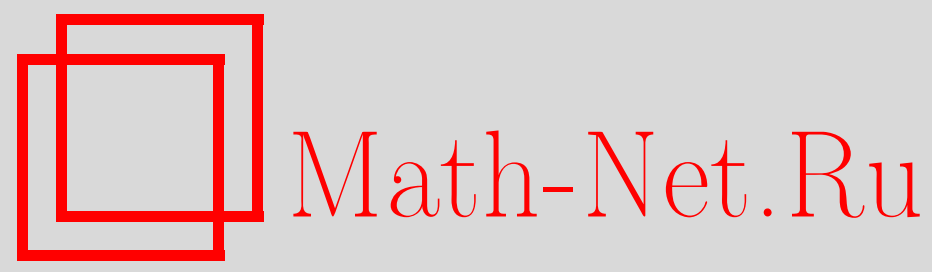

А. Б. Муравник, О задаче Коши для некоторых неоднородных дифференциально-разностных параболических уравнений, Матем. заметки, 2003, том 74, выпуск 4, 538-548

DOI: https://doi.org/10.4213/mzm288

Использование Общероссийского математического портала Math-Net.Ru подразумевает, что вы прочитали и согласны с пользовательским соглашением http://www.mathnet.ru/rus/agreement

Параметры загрузки:

IP : 44.207 .124 .84

26 апреля 2023 г., 16:53:16

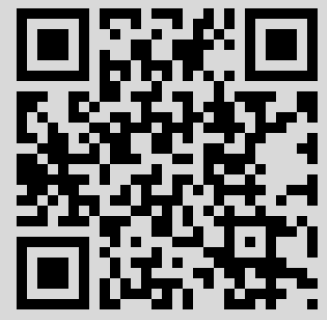




\section{О ЗАДАЧЕ КОШИ ДЛЯ НЕКОТОРЫХ НЕОДНОРОДНЫХ ДИФ ФЕРЕНЦИАЛЬНО-РАЗНОСТНЫХ ПАРАБОЛИЧЕСКИХ УРАВНЕНИЙ}

\section{А. Б. Муравник}

Доказана теорема существования и единственности классического решения задачи Коши для неоднородного уравнения параболического типа, содержащего сдвиги по пространственным переменньм.

Библиографоия: 11 названий.

1. Введение. Параболические уравнения, содержащие операторы сдвига по пространственньг переменным (в том числе абстрактные уравнения в банаховьх и гильбертовых пространствах), исследовались ранее в ряде работ (см., например, [1]-[7] и имеющуюся там библиографию). Вопросы единственности решения задачи Коши для указанных уравнений изучались в [8] (см. также имеющуюся там библиографию).

В работе [9] исследовалась задача Коши для дифференциально-разностного параболического уравнения следующего вида:

$$
\frac{\partial u}{\partial t}=\Delta u+\sum_{k=1}^{m} a_{k} u\left(x-b_{k} h, t\right), \quad x \in \mathbb{R}^{n}, \quad t>0
$$

где $a, b$ - произвольные параметры из $\mathbb{R}^{m}, h$ - фиксированный вектор единичной длины в $\mathbb{R}^{n}$.

В настоящей работе мы рассмотрим неоднородное уравнение. Будет доказана классическая разрешимость задачи Коши, получено интегральное представление решения и установлены классы единственности. Для этого мы используем фундаментальное решение уравнения (1), найденное в [9].

2. Формулировка теоремы существования. Пусть $a, b \in \mathbb{R}^{m}, h$ - фиксированньй вектор единичной длины в $\mathbb{R}^{n}, u_{0}(x)$ непрерьвна и ограничена в $\mathbb{R}^{n}, f(x, t)$ и ее первые производные по пространственньм переменньм непрерьвны и ограничены в $\mathbb{R}^{n} \times(0,+\infty)$. Рассмотрим в $\mathbb{R}^{n} \times(0,+\infty)$ следуюшее уравнение:

$$
\frac{\partial u}{\partial t}-\Delta u-\sum_{k=1}^{m} a_{k} u\left(x-b_{k} h, t\right)=f(x, t) .
$$

Работа выполнена при поддержке фонда ИНТАС, грант № 00-136 и Российского фонда фундаментальных исследований, грант № 02-01-00312. 
Отметим, что уравнения такого вида встречаются, например, в задачах нелинейной оптики (см. [10] и [11]). Наряду с уравнением (2) рассмотрим начальное условие

$$
\left.u\right|_{t=0}=u_{0}(x)
$$

Обозначим через $\mathscr{E}(x, t)$ функцию

$$
\frac{1}{(2 \pi)^{n}} \int_{\mathbb{R}^{n}} \exp \left(-t\left(|\xi|^{2}-\sum_{k=1}^{m} a_{k} \cos \left(b_{k} h\right) \xi\right) \cos \left(x \cdot \xi-t \sum_{k=1}^{m} a_{k} \sin b_{k} h \cdot \xi\right) d \xi\right.
$$

Теперь определим на $\mathbb{R}^{n} \times(0,+\infty)$ следующую функцию:

$$
u(x, t) \stackrel{\text { def }}{=} \int_{\mathbb{R}^{n}} \mathscr{E}(x-y, t) u_{0}(y) d y+\int_{0}^{t} \int_{\mathbb{R}^{n}} \mathscr{E}(x-y, t-\tau) f(y, \tau) d y d \tau .
$$

Справедливо следующее утверждение.

ТЕОрема 1. Пусть $u_{0}(x)$ непрерывна и ограничена в $\mathbb{R}^{n}, f(x, t)$ и ее первые производные по пространственным переменным непрерывны и ограничены в $\mathbb{R}^{n} \times$ $(0,+\infty)$. Тогда функиия, определенная равенством (5), является классическим решением задачи (2), (3).

Доказательству этой теоремы предпошлем следующую лемму.

\section{ЛЕмма 1. Имеет место сходимость}

$$
\int_{0}^{\infty} \exp \left(-z^{2}+t \sum_{k=1}^{m} a_{k} \cos \frac{h_{k} z}{\sqrt{t}}\right) \cos \left(2 z \eta-t \sum_{k=1}^{m} a_{k} \sin \frac{h_{k} z}{\sqrt{t}}\right) d z \stackrel{t \rightarrow+0}{\longrightarrow} \frac{\sqrt{\pi}}{2} e^{-\eta^{2}}
$$

равномерно по $\eta \in \mathbb{R}^{1}$.

ДокАЗАТЕЛЬСТво. Нужно показать, что для любого положительного $\varepsilon$ существует такое положительное $\delta$, что для любого $t \in(0, \delta)$, для любого вещественного $\eta$ вьполнена оценка

$$
\begin{aligned}
& \mid \int_{0}^{\infty} \exp \left(-z^{2}+t \sum_{k=1}^{m} a_{k} \cos \frac{h_{k} z}{\sqrt{t}}\right) \cos \left(2 z \eta-t \sum_{k=1}^{m} a_{k} \sin \frac{h_{k} z}{\sqrt{t}}\right) d z \\
& -\int_{0}^{\infty} e^{-z^{2}} \cos 2 z \eta d z \mid<\varepsilon .
\end{aligned}
$$

Итак, пусть $\varepsilon>0$. Рассмотрим

$$
\begin{aligned}
\int_{0}^{\infty} & e^{-z^{2}}\left[\exp \left(t \sum_{k=1}^{m} a_{k} \cos \frac{h_{k} z}{\sqrt{t}}\right) \cos \left(2 z \eta-t \sum_{k=1}^{m} a_{k} \sin \frac{h_{k} z}{\sqrt{t}}\right)-\cos 2 z \eta\right] d z \\
= & \int_{0}^{\infty} e^{-z^{2}}\left[\operatorname { e x p } ( t \sum _ { k = 1 } ^ { m } a _ { k } \operatorname { c o s } \frac { h _ { k } z } { \sqrt { t } } ) \left(\cos 2 z \eta \cos \left(t \sum_{k=1}^{m} a_{k} \sin \frac{h_{k} z}{\sqrt{t}}\right)\right.\right. \\
& \left.\left.-\sin 2 z \eta \sin \left(t \sum_{k=1}^{m} a_{k} \sin \frac{h_{k} z}{\sqrt{t}}\right)\right)-\cos 2 z \eta\right] d z .
\end{aligned}
$$


Выберем $\delta$ из $(0,1]$ настолько малым, что синус монотонен на промежутке

$$
\left(-\delta \sum_{k=1}^{m}\left|a_{k}\right|, \delta \sum_{k=1}^{m}\left|a_{k}\right|\right)
$$

и

$$
\sin \left(\delta \sum_{k=1}^{m}\left|a_{k}\right|\right)<\frac{\varepsilon}{2}\left(\exp \left(t \sum_{k=1}^{m}\left|a_{k}\right|\right) \int_{0}^{\infty} e^{-z^{2}} d z\right)^{-1} \quad \text { для любого } t \in(-\delta, \delta) .
$$

Тогда при $t \in(0, \delta)$

$$
\begin{aligned}
& \left|\int_{0}^{\infty} e^{-z^{2}} \exp \left(t \sum_{k=1}^{m} a_{k} \cos \frac{h_{k} z}{\sqrt{t}}\right) \sin 2 z \eta \sin \left(t \sum_{k=1}^{m} a_{k} \sin \frac{h_{k} z}{\sqrt{t}}\right) d z\right| \\
& \quad \leqslant \frac{\varepsilon}{2}\left(\exp \left(t \sum_{k=1}^{m}\left|a_{k}\right|\right) \int_{0}^{\infty} e^{-z^{2}} d z\right)^{-1} \int_{0}^{\infty} e^{-z^{2}} \exp \left(t \sum_{k=1}^{m}\left|a_{k}\right|\right) d z=\frac{\varepsilon}{2} .
\end{aligned}
$$

Осталось показать, что это (достаточно малое) $\delta$ можно выбрать так, что для любых вещественньх $z, \eta$ при $t \in(0, \delta)$ вьполняется еще и следующее неравенство:

$$
|\cos 2 z \eta|\left|\exp \left(t \sum_{k=1}^{m} a_{k} \cos \frac{h_{k} z}{\sqrt{t}}\right) \cos \left(t \sum_{k=1}^{m} a_{k} \sin \frac{h_{k} z}{\sqrt{t}}\right)-1\right| \leqslant \frac{\varepsilon}{2}\left(\int_{0}^{\infty} e^{-z^{2}} d z\right)^{-1} .
$$

То есть достаточно показать, что

$$
\exp \left(t \sum_{k=1}^{m} a_{k} \cos \frac{h_{k} z}{\sqrt{t}}\right) \cos \left(t \sum_{k=1}^{m} a_{k} \sin \frac{h_{k} z}{\sqrt{t}}\right) \stackrel{t \rightarrow+0}{\longrightarrow} 1
$$

равномерно по $z \in \mathbb{R}^{1}$. Имеем

$$
\begin{aligned}
& \exp \left(t \sum_{k=1}^{m} a_{k} \cos \frac{h_{k} z}{\sqrt{t}}\right) \cos \left(t \sum_{k=1}^{m} a_{k} \sin \frac{h_{k} z}{\sqrt{t}}\right)-1 \\
& =\exp \left(t \sum_{k=1}^{m} a_{k} \cos \frac{h_{k} z}{\sqrt{t}}\right)\left[1-2 \sin ^{2}\left(\frac{t}{2} \sum_{k=1}^{m} a_{k} \sin \frac{h_{k} z}{\sqrt{t}}\right)\right]-1 \\
& \quad=\exp \left(t \sum_{k=1}^{m} a_{k} \cos \frac{h_{k} z}{\sqrt{t}}\right)-1-2 \exp \left(t \sum_{k=1}^{m} a_{k} \cos \frac{h_{k} z}{\sqrt{t}}\right) \sin ^{2}\left(\frac{t}{2} \sum_{k=1}^{m} a_{k} \sin \frac{h_{k} z}{\sqrt{t}}\right)
\end{aligned}
$$

Выберем $t$ настолько малым, что

$$
\sin ^{2}\left(\frac{t}{2} \sum_{k=1}^{m}\left|a_{k}\right|\right) \leqslant \frac{\varepsilon}{8} \exp \left(-\sum_{k=1}^{m}\left|a_{k}\right|\right)
$$

В силу монотонности синуса в достаточно малой положительной полуокрестности начала координат

$$
2 \sin ^{2}\left(\frac{t}{2} \sum_{k=1}^{m} a_{k} \sin \frac{h_{k} z}{\sqrt{t}}\right) \leqslant \frac{\varepsilon}{4} \exp \left(-\sum_{k=1}^{m}\left|a_{k}\right|\right)
$$


для указанного $t$. Без ограничения общности можно считать, что выбранное $t$ принадлежит промежутку $(0,1)$, следовательно, для любого вешественного $z$

$$
2 \sin ^{2}\left(\frac{t}{2} \sum_{k=1}^{m} a_{k} \sin \frac{h_{k} z}{\sqrt{t}}\right) \exp \left(t \sum_{k=1}^{m} a_{k} \cos \frac{h_{k} z}{\sqrt{t}}\right) \leqslant \frac{\varepsilon}{4}
$$

Итак, осталось оценить $\exp \left(t \sum_{k=1}^{m} a_{k} \cos \left(h_{k} z / \sqrt{t}\right)\right)-1$.

Без ограничения общности можно считать, что $t$ настолько мало, что

$$
1-\frac{\varepsilon}{4}<\exp \left(-\sum_{k=1}^{m}\left|a_{k}\right| t\right)<\exp \left(\sum_{k=1}^{m}\left|a_{k}\right| t\right)<1+\frac{\varepsilon}{4}
$$

Следовательно,

$$
1-\frac{\varepsilon}{4}<\exp \left(-\sum_{k=1}^{m}\left|a_{k}\right| t\right)<\exp \left(t \sum_{k=1}^{m} a_{k} \cos \frac{h_{k} z}{\sqrt{t}}\right)<\exp \left(\sum_{k=1}^{m}\left|a_{k}\right| t\right)<1+\frac{\varepsilon}{4}
$$

для любого $z \in \mathbb{R}^{1}$.

Отсюда

$$
\left|\exp \left(t \sum_{k=1}^{m} a_{k} \cos \frac{h_{k} z}{\sqrt{t}}\right)-1\right|<\frac{\varepsilon}{4}
$$

для любого $z \in \mathbb{R}^{1}$.

3. Доказательство теоремы существования. Введем следуюшие обозначения:

$$
u_{1}(x, t) \stackrel{\text { def }}{=} \int_{\mathbb{R}^{n}} \mathscr{E}(x-y, t) u_{0}(y) d y, \quad u_{2}(x, t) \stackrel{\text { def }}{=} \int_{0}^{t} \int_{\mathbb{R}^{n}} \mathscr{E}(x-y, t-\tau) f(y, \tau) d y .
$$

Как известно из [9], функция $u_{1}(x, t)$ является классическим решением задачи $(1),(3)$. Поэтому достаточно доказать, что функция $u_{2}(x, t)$ удовлетворяет (в классическом смысле) уравнению (2) и $\left.u_{2}\right|_{t=0} \equiv 0$.

Для доказательства первого из этих утверждений заметим (см. [9]), что $\mathscr{E}(x, t)$ удовлетворяет уравнению $(1)$ в $\mathbb{R}^{n} \times(0,+\infty)$ и для любого фиксированного $t$ стремится $\mathrm{K}$ нулю (вместе со всеми своими производными любого порядка) при $x \rightarrow \infty$ быстрее любой отрицательной степени $|x|$. Поэтому остается доказать следующую лемму.

Лемма 2. Пусть $x_{0} \in \mathbb{R}^{n}, t_{0}>0$. Тогда

$$
\lim _{\tau \rightarrow+0} \int_{\mathbb{R}^{n}} f\left(\xi, t_{0}-\tau\right) \mathscr{E}\left(x_{0}-\xi, \tau\right) d \xi=f\left(x_{0}, t_{0}\right)
$$


ДокАЗАТЕЛЬСтво. Прежде всего введем следующую функцию двух переменных:

$$
\mathscr{E}_{0}(x, t) \stackrel{\text { def }}{=} \frac{1}{\pi} \int_{0}^{\infty} \exp \left(-t\left(\xi^{2}-\sum_{k=1}^{m} a_{k} \cos b_{k} \xi\right)\right) \cos \left(x \xi-t \sum_{k=1}^{m} a_{k} \sin b_{k} \xi\right) d \xi .
$$

Далее, без ограничения общности (поскольку оператор Лапласа инвариантен относительно поворота) можно считать, что вектор $h$ совпадает по направлению с первой координатной осью, т.е. что $h=(1,0, \ldots, 0)$. Это позволяет представить функцию $(2 \pi)^{n} \mathscr{E}(x, t)$ в виде

$$
\begin{aligned}
& \int_{\mathbb{R}^{n}} \exp \left(t\left(\sum_{k=1}^{m} a_{k} \cos b_{k} \xi_{1}-|\xi|^{2}\right)\right) \cos \left(x_{1} \xi_{1}+\cdots+x_{n} \xi_{n}-t \sum_{k=1}^{m} a_{k} \sin b_{k} \xi_{1}\right) d \xi \\
& =\int_{\mathbb{R}^{n}} \exp \left(t\left(\sum_{k=1}^{m} a_{k} \cos b_{k} \xi_{1}-\xi_{1}^{2}\right)\right) e^{-t\left|\xi^{\prime}\right|^{2}} \cos \left(x_{1} \xi_{1}-t \sum_{k=1}^{m} a_{k} \sin b_{k} \xi_{1}\right) \\
& \times \cos x^{\prime} \cdot \xi^{\prime} d \xi-\int_{\mathbb{R}^{n}} \exp \left(t\left(\sum_{k=1}^{m} a_{k} \cos b_{k} \xi_{1}-\xi_{1}^{2}\right)\right) e^{-t\left|\xi^{\prime}\right|^{2}} \\
& \times \sin \left(x_{1} \xi_{1}-t \sum_{k=1}^{m} a_{k} \sin b_{k} \xi_{1}\right) \sin x^{\prime} \cdot \xi^{\prime} d \xi \\
& =\int_{-\infty}^{\infty} \exp \left(t\left(\sum_{k=1}^{m} a_{k} \cos b_{k} \xi_{1}-\xi_{1}^{2}\right)\right) \cos \left(x_{1} \xi_{1}-t \sum_{k=1}^{m} a_{k} \sin b_{k} \xi_{1}\right) d \xi_{1} \\
& \times \int_{\mathbb{R}^{n-1}} e^{-t\left|\xi^{\prime}\right|^{2}} \cos x^{\prime} \cdot \xi^{\prime} d \xi^{\prime}-\int_{-\infty}^{\infty} \exp \left(t\left(\sum_{k=1}^{m} a_{k} \cos b_{k} \xi_{1}-\xi_{1}^{2}\right)\right) \\
& \times \sin \left(x_{1} \xi_{1}-t \sum_{k=1}^{m} a_{k} \sin b_{k} \xi_{1}\right) d \xi_{1} \int_{\mathbb{R}^{n-1}} e^{-t\left|\xi^{\prime}\right|^{2}} \sin x^{\prime} \cdot \xi^{\prime} d \xi^{\prime} \\
& =2 \pi \mathscr{E}_{0}\left(x_{1}, t\right) \int_{\mathbb{R}^{n-1}} e^{-t\left|\xi^{\prime}\right|^{2}} \cos x^{\prime} \cdot \xi^{\prime} d \xi^{\prime} .
\end{aligned}
$$

Вычислим последний интеграл. Без ограничения общности, а именно, с точностью до поворота координатной системы $\xi_{1}, \ldots, \xi_{n}$ (якобиан такой замены переменных равен единище), $x^{\prime} \cdot \xi^{\prime}=|x| \xi_{2}$. Поэтому,

$$
\begin{aligned}
\int_{\mathbb{R}^{n-1}} & e^{-t\left|\xi^{\prime}\right|^{2}} \cos x^{\prime} \cdot \xi^{\prime} d \xi^{\prime}=\int_{\mathbb{R}^{n-1}} e^{-t\left|\xi^{\prime}\right|^{2}} \cos \left|x^{\prime}\right| \xi_{2} d \xi \\
= & \int_{-\infty}^{+\infty} e^{-t \xi_{2}^{2}} \cos \left|x^{\prime}\right| \xi_{2} d \xi_{2} \int_{\mathbb{R}^{n-2}} e^{-t\left|\xi^{\prime \prime}\right|^{2}} d \xi^{\prime \prime} \\
= & \frac{\sqrt{\pi}}{\sqrt{t}} e^{-\left|x^{\prime}\right|^{2} /(4 t)} \int_{\mathbb{R}^{n-2}} e^{-t\left(\xi_{3}^{2}+\cdots+\xi_{n}^{2}\right)} d \xi^{\prime \prime}=\left(\frac{\pi}{t}\right)^{(n-1) / 2} e^{-\left|x^{\prime}\right|^{2} /(4 t)} .
\end{aligned}
$$

Итак,

$$
\mathscr{E}(x, t)=\frac{1}{(2 \sqrt{\pi t})^{n-1}} \mathscr{E}_{0}\left(x_{1}, t\right) e^{-\left|x^{\prime}\right|^{2} /(4 t)},
$$

где $x^{\prime}=\left(x_{2}, \ldots, x_{n}\right) \in \mathbb{R}^{n-1}$. 
Переобозначим $x_{0}$ через $\left(y_{0}, x_{1}^{0}, \ldots, x_{n-1}^{0}\right) \stackrel{\text { def }}{=}\left(y_{0}, x^{0}\right)$ и рассмотрим

$$
F(\tau) \stackrel{\text { def }}{=} \int_{\mathbb{R}^{n}} f\left(\xi, t_{0}-\tau\right) \mathscr{E}\left(x_{0}-\xi, \tau\right) d \xi .
$$

Используя полученное выше представление $\mathscr{E}$ через $\mathscr{E}_{0}$ и замену переменных

$$
\frac{y_{0}-\xi_{1}}{2 \sqrt{\tau}}=\eta, \quad \frac{x_{j}^{0}-\xi_{j+1}}{2 \sqrt{\tau}}=z_{j}, \quad j=\overline{1, n-1},
$$

получаем

$$
\begin{aligned}
F(\tau)= & \frac{2}{\pi^{(n-1) / 2}} \int_{\mathbb{R}^{n}} \sqrt{\tau} \mathscr{E}_{0}(2 \sqrt{\tau} \eta, \tau) e^{-|z|^{2}} \\
& \times f\left(y_{0}-2 \sqrt{\tau} \eta, x_{1}^{0}-2 \sqrt{\tau} z_{1}, \ldots, x_{n-1}^{0}-2 \sqrt{\tau} z_{n-1}, t_{0}-\tau\right) d \eta d z \\
= & \frac{2}{\pi^{(n-1) / 2}} \int_{-\infty}^{+\infty} \int_{\mathbb{R}^{n-1}} \sqrt{\tau} \mathscr{E}_{0}(2 \sqrt{\tau} \eta, \tau) e^{-|\xi|^{2}} \\
& \times f\left(y_{0}-2 \sqrt{\tau} \eta, x^{0}-2 \sqrt{\tau} \xi, t_{0}-\tau\right) d \xi d \eta
\end{aligned}
$$

(для удобства возвращаемся к традиционным обозначениям переменньх интегрирования),

$$
f\left(x_{0}, t_{0}\right)=\frac{1}{\pi^{n / 2}} \int_{-\infty}^{+\infty} \int_{\mathbb{R}^{n-1}} e^{-\eta^{2}-|\xi|^{2}} f\left(y_{0}, x^{0}, t_{0}\right) d \xi d \eta .
$$

Следовательно,

$$
\begin{aligned}
F(\tau)-f\left(x_{0}, t_{0}\right)= & \frac{2}{\pi^{(n+1) / 2}} \int_{-\infty}^{+\infty} \int_{\mathbb{R}^{n-1}} e^{-|\xi|^{2}}\left[\pi \sqrt{\tau} \mathscr{E}_{0}(2 \sqrt{\tau} \eta, \tau)\right. \\
& \left.\times f\left(y_{0}-2 \sqrt{\tau} \eta, x^{0}-2 \sqrt{\tau} \xi, t_{0}-\tau\right)-\frac{\sqrt{\pi}}{2} f\left(y_{0}, x^{0}, t_{0}\right) e^{-\eta^{2}}\right] d \xi d \eta
\end{aligned}
$$

Далее,

$$
\begin{aligned}
\pi \sqrt{\tau} \mathscr{E}_{0}(2 \sqrt{\tau} \eta, \tau)= & \sqrt{\tau} \int_{0}^{\infty} \exp \left(-\tau\left(\xi^{2}-\sum_{k=1}^{m} a_{k} \cos b_{k} \xi\right)\right) \\
& \times \cos \left(2 \sqrt{\tau} \eta \xi-\tau \sum_{k=1}^{m} a_{k} \sin b_{k} \xi\right) d \xi \\
= & \int_{0}^{\infty} \exp \left(-z^{2}+\tau \sum_{k=1}^{m} a_{k} \cos \frac{b_{k} z}{\sqrt{\tau}}\right) \\
& \times \cos \left(2 z \eta-\tau \sum_{k=1}^{m} a_{k} \sin \frac{b_{k} z}{\sqrt{\tau}}\right) d z .
\end{aligned}
$$

Значит,

$$
\begin{aligned}
F(\tau) & -f\left(x_{0}, t_{0}\right)=\frac{2}{\pi^{\frac{n+1}{2}}} \int_{-\infty}^{+\infty} \int_{\mathbb{R}^{n-1}} e^{-|\xi|^{2}}\left[f\left(y_{0}-2 \sqrt{\tau} \eta, x^{0}-2 \sqrt{\tau} \xi, t_{0}-\tau\right)\right. \\
& \times \int_{0}^{\infty} \exp \left(-z^{2}+\tau \sum_{k=1}^{m} a_{k} \cos \frac{b_{k} z}{\sqrt{\tau}}\right) \cos \left(2 z \eta-\tau \sum_{k=1}^{m} a_{k} \sin \frac{b_{k} z}{\sqrt{\tau}}\right) d z \\
& \left.-\frac{\sqrt{\pi}}{2} f\left(y_{0}, x^{0}, t_{0}\right) e^{-\eta^{2}}\right] d \xi d \eta
\end{aligned}
$$


Пусть теперь

$$
A>0, \quad G_{1}=\{(\eta, \xi)|\eta \in(-A, A),| \xi \mid<A\}, \quad G_{2}=\mathbb{R}^{n} \backslash G_{1} .
$$

Тогда $F(\tau)-f\left(x_{0}, t_{0}\right)=J_{1}+J_{2}$, где

$$
\begin{aligned}
J_{j}= & \frac{2}{\pi^{(n+1) / 2}} \int_{G_{j}}\left[\int_{0}^{\infty} f\left(y_{0}-2 \sqrt{\tau} \eta, x^{0}-2 \sqrt{\tau} \xi, t_{0}-\tau\right) \exp \left(-z^{2}+\tau \sum_{k=1}^{m} a_{k} \cos \frac{b_{k} z}{\sqrt{\tau}}\right)\right. \\
& \left.\times \cos \left(2 z \eta-\tau \sum_{k=1}^{m} a_{k} \sin \frac{b_{k} z}{\sqrt{\tau}}\right) d z-\frac{\sqrt{\pi}}{2} f\left(y_{0}, x^{0}, t_{0}\right) e^{-\eta^{2}}\right] e^{-|\xi|^{2}} d \eta d \xi, \quad j=1,2 .
\end{aligned}
$$

Сначала оценим интеграл $J_{2}$. Рассмотрим для этого

$$
\begin{aligned}
\left|\int_{G_{2}} f\left(y_{0}, x^{0}, t_{0}\right) e^{-|\xi|^{2}-\eta^{2}} d \eta d \xi\right| & \leqslant \sup |f| \int_{|\xi|^{2}+\eta^{2} \geqslant A^{2}} e^{-|\xi|^{2}-\eta^{2}} d \xi d \eta \\
& =C_{n} \sup |f| \int_{A}^{\infty} r^{n-1} e^{-r^{2}} d r \stackrel{A \rightarrow \infty}{\longrightarrow} 0
\end{aligned}
$$

(в силу сходимости последнего интеграла и ограниченности $f$ ).

Для оценки оставшегося слагаемого интеграла $J_{2}$ разобьем область интегрирования следуюшим образом:

$$
\begin{aligned}
G_{2} & =\left\{\eta>A, \xi \in \mathbb{R}^{n-1}\right\} \cup\left\{\eta<-A, \xi \in \mathbb{R}^{n-1}\right\} \cup\{\eta \in[-A, A],|\xi| \geqslant A\} \\
& \stackrel{\text { def }}{=} G_{2,1} \cup G_{2,2} \cup G_{2,3}
\end{aligned}
$$

соответственно для $j=1,2,3$ обозначим

$$
\begin{gathered}
\int_{G_{2, j}} e^{-|\xi|^{2}} \int_{0}^{\infty} f\left(y_{0}-2 \sqrt{\tau} \eta, x^{0}-2 \sqrt{\tau} \xi, t_{0}-\tau\right) \exp \left(-z^{2}+\tau \sum_{k=1}^{m} a_{k} \cos \frac{b_{k} z}{\sqrt{t}}\right) \\
\times \cos \left(2 z \eta-\tau \sum_{k=1}^{m} a_{k} \sin \frac{b_{k} z}{\sqrt{\tau}}\right) d z d \eta d \xi \quad \text { через } \frac{\pi^{(n+1) / 2}}{2} J_{2, j} .
\end{gathered}
$$

Тогда

$$
\begin{aligned}
J_{2,1}=\frac{2}{\pi^{(n+1) / 2}} \int_{G_{2,1}} e^{-|\xi|^{2}} u_{0}\left(y_{0}-2 \sqrt{\tau} \eta, x^{0}-2 \sqrt{\tau} \xi, t_{0}-\tau\right) \\
\quad \times \int_{0}^{\infty} \exp \left(-z^{2}+\tau \sum_{k=1}^{m} a_{k} \cos \frac{b_{k} z}{\sqrt{\tau}}\right) \cos 2 z \eta \\
\quad \times \cos \left(\tau \sum_{k=1}^{m} a_{k} \sin \frac{b_{k} z}{\sqrt{\tau}}\right) d z d \eta d \xi \\
+\frac{2}{\pi^{(n+1) / 2}} \int_{G_{2,1}} f\left(y_{0}-2 \sqrt{\tau} \eta, x^{0}-2 \sqrt{\tau} \xi, t_{0}-\tau\right) \\
\quad \times \int_{0}^{\infty} \exp \left(-z^{2}+\tau \sum_{k=1}^{m} a_{k} \cos \frac{b_{k} z}{\sqrt{\tau}}\right) \sin 2 z \eta \\
\quad \times \sin \left(\tau \sum_{k=1}^{m} a_{k} \sin \frac{b_{k} z}{\sqrt{\tau}}\right) d z e^{-|\xi|^{2}} d \eta d \xi \stackrel{\text { def }}{=} J_{2,1,1}+J_{2,1,2} .
\end{aligned}
$$


После двукратного интегрирования по частям

$$
\int_{0}^{\infty} \exp \left(-z^{2}+\tau \sum_{k=1}^{m} a_{k} \cos \frac{b_{k} z}{\sqrt{\tau}}\right) \cos 2 z \eta \cos \left(\tau \sum_{k=1}^{m} a_{k} \sin \frac{b_{k} z}{\sqrt{\tau}}\right) d z
$$

принимает вид

$$
-\frac{1}{4 \eta^{2}} \int_{0}^{\infty}\left[\exp \left(-z^{2}+\tau \sum_{k=1}^{m} a_{k} \cos \frac{b_{k} z}{\sqrt{\tau}}\right) \cos \left(\tau \sum_{k=1}^{m} a_{k} \sin \frac{b_{k} z}{\sqrt{\tau}}\right)\right]_{z}^{\prime \prime} \cos 2 z \eta d z
$$

(с учетом того, что $|\eta| \geqslant A>0$ в области $G_{2,1}$ ).

Считая (без ограничения общности), что $\tau \leqslant 1$, получаем (при помощи непосредственного дифференцирования), что в (7) абсолютная величина интеграла не превосходит константы, зависящей только от параметров $a$ и $b$. Отсюда,

$$
\left|J_{2,1,1}\right| \leqslant M \sup |f| \int_{\mathbb{R}^{n-1}} e^{-|\xi|^{2}} d \xi \int_{A}^{\infty} \frac{d \eta}{\eta^{2}}=\frac{M \pi^{(n-1) / 2} \sup |f|}{A} .
$$

Аналогично оценивается $J_{2,1,2}$. Отсюда, $\left|J_{2,1}\right| \leqslant 2 M \pi^{(n-1) / 2} \sup |f| / A$.

Точно так же получаем, что $\left|J_{2,2}\right| \leqslant 2 M \pi^{(n-1) / 2} \sup |f| /(\pi A)$.

Теперь оценим

$$
\begin{aligned}
J_{2,3}= & \frac{2}{\pi^{(n+1) / 2}} \int_{|\xi| \geqslant A} e^{-|\xi|^{2}} \int_{-A}^{A} f\left(y_{0}-2 \sqrt{\tau} \eta, x^{0}-2 \sqrt{\tau} \xi, t_{0}-\tau\right) \\
& \times \int_{0}^{\infty} \exp \left(-z^{2}+\tau \sum_{k=1}^{m} a_{k} \cos \frac{b_{k} z}{\sqrt{\tau}}\right) \cos 2 z \eta \cos \left(\tau \sum_{k=1}^{m} a_{k} \sin \frac{b_{k} z}{\sqrt{\tau}}\right) d z d \eta d \xi
\end{aligned}
$$

при $\tau \in(0,1]$ имеем

$$
\begin{gathered}
\left|\int_{0}^{\infty} \exp \left(-z^{2}+\tau \sum_{k=1}^{m} a_{k} \cos \frac{b_{k} z}{\sqrt{\tau}}\right) \cos 2 z \eta \cos \left(\tau \sum_{k=1}^{m} a_{k} \sin \frac{b_{k} z}{\sqrt{\tau}}\right) d z\right| \\
\leqslant e^{\tau} \int_{0}^{\infty} e^{-z^{2}} d z \leqslant \frac{\sqrt{\pi}}{2} e
\end{gathered}
$$

Отсюда

$$
\left|J_{2,3}\right| \leqslant \frac{2 \sup |f| e}{\pi^{n / 2}} A \int_{|\xi| \geqslant A} e^{-|\xi|^{2}} d \xi=\frac{2 C_{n} \sup |f| e}{\pi^{n / 2}} A \int_{A}^{\infty} r^{n-2} e^{-r^{2}} d r .
$$

Последнее выражение стремится к нулю при $A \rightarrow \infty$. Действительно,

$$
x \int_{x}^{\infty} r^{n-2} e^{-r^{2}} d r=x\left(\frac{\Gamma(n / 2)}{\sqrt{2}}-\int_{0}^{x} r^{n-2} e^{-r^{2}} d r\right)=\frac{\Gamma(n / 2) / \sqrt{2}-\int_{0}^{x} r^{n-2} e^{-r^{2}} d r}{1 / x} .
$$

\section{Вычисляя}

$$
\lim _{x \rightarrow \infty} \frac{\Gamma(n / 2) / \sqrt{2}-\int_{0}^{x} r^{n-2} e^{-r^{2}} d r}{1 / x}
$$

по правилу Лопиталя, получаем, что он равен $\lim _{x \rightarrow \infty} x^{n} e^{-x^{2}}=0$. 
Таким образом, для произвольного положительного $\varepsilon$ можно выбрать такое $A \in$ $(0,+\infty)$, что $\left|J_{2}\right|<\varepsilon / 2$; зафиксируем такое $A$ и рассмотрим интеграл $J_{1}$.

В силу непрерьвности функции $f$ в точке $\left(y_{0}, x^{0}, t_{0}\right)$ и леммы 1 можно выбрать положительное $\tau_{0}$ настолько малым, что для любого $\tau$ из $\left(0, \tau_{0}\right)$, для любого $(\eta, \xi)$ из $G_{1}$

$$
\begin{aligned}
& \mid \int_{0}^{\infty} \exp \left(-z^{2}+\tau \sum_{k=1}^{m} a_{k} \cos \frac{b_{k} z}{\sqrt{\tau}}\right) \cos \left(2 z \eta-\tau \sum_{k=1}^{m} a_{k} \sin \frac{b_{k} z}{\sqrt{\tau}}\right) d z \\
& \quad \times f\left(y_{0}-2 \sqrt{\tau} \eta, x^{0}-2 \sqrt{\tau} \xi, t_{0}-\tau\right)-f\left(y_{0}, x^{0}, t_{0}\right) \frac{\sqrt{\pi}}{2} e^{-\eta^{2}} \mid<\frac{\varepsilon}{2} \frac{\pi^{(n+1) / 2}}{2} \frac{1}{(2 A)^{n}},
\end{aligned}
$$

следовательно, $\left|J_{1}\right|<\varepsilon / 2$.

Итак, $u_{2}(x, t)$ удовлетворяет уравнению (2). Теперь докажем следующее утверждение.

Лемма 3. Пусть $x_{0} \in \mathbb{R}^{n}$. Тогда $u_{2}\left(x_{0}, t\right) \stackrel{t \rightarrow+0}{\longrightarrow} 0$.

ДоКАЗАТЕЛЬСТВО. Рассмотрим функцию

$$
\int_{\mathbb{R}^{n}} \mathscr{E}\left(x_{0}-y, \tau\right) f(y, t-\tau) d y
$$

определенную при $t \in(0,+\infty), \tau \in(0, t)$.

Представляя $\mathscr{E}$ через $\mathscr{E}_{0}$, получаем (см. доказательство леммы 2$)$, что последний интеграл равен

$$
\begin{aligned}
& \frac{2}{\pi^{(n+1) / 2}} \int_{-\infty}^{+\infty} \int_{\mathbb{R}^{n-1}} e^{-\left|\eta^{\prime}\right|^{2}} f\left(x_{0}-2 \sqrt{t} \eta, t-\tau\right) \int_{0}^{\infty} \exp \left(-z^{2}+\tau \sum_{k=1}^{m} a_{k} \cos \frac{b_{k} z}{\sqrt{\tau}}\right) \\
& \quad \times \cos \left(2 z \eta_{n}-\tau \sum_{k=1}^{m} a_{k} \sin \frac{b_{k} z}{\sqrt{\tau}}\right) d z d \eta \stackrel{\text { def }}{=} \frac{2}{\pi^{(n+1) / 2}} G(t, \tau) .
\end{aligned}
$$

Докажем ограниченность $G(t, \tau)$ в некоторой (достаточно малой) положительной полуокрестности точки $t=0$. Для этого разобьем $G(t, \tau)$ на слагаемые следующим образом:

$$
G(t, \tau)=\int_{G_{1}}+\int_{G_{2}} \stackrel{\text { def }}{=} J_{1}+J_{2}
$$

где

$$
G_{1}=\left\{\eta\left|\eta_{n} \in(-1,1),\right| \eta^{\prime} \mid<1\right\}, \quad G_{2}=\mathbb{R}^{n} \backslash G_{1} .
$$

Для оценки слагаемого $J_{2}$ разобьем $G_{2}$ на области

$$
\left\{\eta_{n}>1, \eta^{\prime} \in \mathbb{R}^{n-1}\right\}, \quad\left\{\eta_{n}<-1, \eta^{\prime} \in \mathbb{R}^{n-1}\right\}, \quad\left\{\eta_{n} \in[-1,1],\left|\eta^{\prime}\right| \geqslant 1\right\}
$$

(обозначим эти области через $G_{2,1}, G_{2,2}, G_{2,3}$, а соответствуюшие интегралы - через $\left.J_{2,1}, J_{2,2}, J_{2,3}\right)$.

Оценивая $J_{2,1}$ и $J_{2,2}$, воспользуемся установленным при доказательстве леммы 2 неравенством (справедливьм при $0<\tau \leqslant 1,\left|\eta_{n}\right|>1$ )

$$
\left|\int_{0}^{\infty} \exp \left(-z^{2}+\tau \sum_{k=1}^{m} a_{k} \cos \frac{b_{k} z}{\sqrt{\tau}}\right) \cos \left(2 z \eta_{n}-\tau \sum_{k=1}^{m} a_{k} \sin \frac{b_{k} z}{\sqrt{\tau}}\right) d z\right|<\frac{M}{\eta_{n}^{2}},
$$

где $M$ зависит только от $a$ и $b$. 
Получим, что

$$
\left|J_{2,1}\right| \leqslant M \sup |f| \int_{1}^{+\infty} \int_{\mathbb{R}^{n-1}} \frac{e^{-\left|\eta^{\prime}\right|^{2}}}{\eta_{n}^{2}} d \eta^{\prime} d \eta_{n}=M \sup |f| \int_{\mathbb{R}^{n-1}} e^{-\left|\eta^{\prime}\right|^{2}} d \eta^{\prime},
$$

т.е. $J_{2,1}$ ограничен; аналогично доказьвается ограниченность $J_{2,2}$. Оценивая $J_{2,3}$, воспользуемся очевидным неравенством

$$
\begin{gathered}
\left|\int_{0}^{\infty} \exp \left(-z^{2}+\tau \sum_{k=1}^{m} a_{k} \cos \frac{b_{k} z}{\sqrt{\tau}}\right) \cos \left(2 z \eta_{n}-\tau \sum_{k=1}^{m} a_{k} \sin \frac{b_{k} z}{\sqrt{\tau}}\right) d z\right| \\
\quad<\frac{\exp \left(|\tau| \sum_{k=1}^{m}\left|a_{k}\right|\right) \sqrt{\pi}}{2}
\end{gathered}
$$

что при $0<\tau \leqslant 1$ дает

$$
\left|J_{2,3}\right| \leqslant \frac{\exp \left(\sum_{k=1}^{m}\left|a_{k}\right|\right) \sqrt{\pi}}{2} \sup |f| \int_{-1}^{1} \int_{\mathbb{R}^{n-1}} e^{-\left|\eta^{\prime}\right|^{2}} d \eta^{\prime} d \eta_{n}=M_{1} \int_{\mathbb{R}^{n-1}} e^{-\left|\eta^{\prime}\right|^{2}} d \eta^{\prime}
$$

(здесь $M_{1}$ зависит только от $a$ и $f$ ), т.е. $J_{2,3}$ ограничен.

Таким образом, $J_{2}$ ограничен. А $J_{1}$ ограничен в силу ограниченности подынтегральной функции (в любой окрестности точки $t=0$ ) и области интегрирования. Таким образом, существует такое положительное $t_{0}$, что $G(t, \tau)$ ограничена при $t \in\left(0, t_{0}\right)$. Значит,

$$
\left|u_{2}\left(x_{0}, t\right)\right| \leqslant \sup _{\left(0, t_{0}\right)}|G| t
$$

для любого $t$ из $\left(0, t_{0}\right)$.

В силу произвольности выбора точки $x_{0}$ теорема 1 доказана полностью.

4. Теорема единственности. Вьполняя двойное дифференцирование в выражении (7), получаем, что для любого положительного $T$ выполнено неравенство

$$
\begin{gathered}
\left|\int_{0}^{\infty} \exp \left(-z^{2}+\tau \sum_{k=1}^{m} a_{k} \cos \frac{b_{k} z}{\sqrt{\tau}}\right) \cos \left(2 z \eta-\tau \sum_{k=1}^{m} a_{k} \sin \frac{b_{k} z}{\sqrt{\tau}}\right) d z\right| \\
<\frac{M(1+T) \exp \left(T \sum_{k=1}^{m}\left|a_{k}\right|\right)}{\eta^{2}}
\end{gathered}
$$

при $0<\tau \leqslant T$, причем $M$ зависит только от $a$ и $b$. Отсюда и из неравенства (8), верного при любом $\tau$, вытекает, что функция (5) ограничена в любой полосе $\mathbb{R}^{n} \times[0, T]$ (см. доказательство леммы 3).

Далее, уравнение (1) можно представить в виде

$$
\frac{\partial u}{\partial t}=\sum_{r=1}^{m+1} L_{r} P_{r} u
$$

где $P_{r}=a_{r} I$ при $r=\overline{1, m}, P_{m+1}=\Delta, L_{m+1}=I$, а при $r=\overline{1, m}$ операторы $L_{r}$ действуют следуюшим образом: $L_{r} g(x)=g\left(x-b_{r} h\right)$. А из [8] известно, что для таких уравнений задача Коши в любой полосе $\mathbb{R}^{n} \times[0, T]$ в случае тривиальной начальной функции не имеет нетривиальных ограниченных решений. Тем самьм, в силу линейности уравнения (2) доказана следующая 
ТЕОрема 2. Пусть $u_{0}(x)$ непрерывна и ограничена в $\mathbb{R}^{n}, f(x, t)$ и ее первые производные по пространственным переменным непрерывны и ограничены в $\mathbb{R}^{n} \times$ $[0,+\infty)$. Тогда функиия, определенная равенством (5), является единственным решением задачи (2), (3), которое ограничено в $\mathbb{R}^{n} \times[0, T]$ при каждом $T>0$.

ЗАмЕЧАНИЕ. Мы использовали единственность ограниченного решения задачи (1), (3), однако в работе [8] доказано и более сильное утверждение. Поэтому решение (5) единственно и в более широком классе, а именно - в классе функций, при любом положительном $T$ удовлетворяющих следующей оценке:

$$
\sup _{t \in[0, T]}|u(x, t)| \leqslant C e^{q|x| \ln (|x|+1)}, \quad \text { если } q<\frac{1}{\max _{1 \leqslant k \leqslant m}\left|b_{k}\right|} .
$$

Автор выражает глубокую признательность А. Л. Скубачевскому за ценные советы и постоянное внимание к работе.

\section{СПИСОК ЦИТИРОВАННОЙ ЛИТЕРАТУРЫ}

[1] Kunisch K., Schappacher W. Necessary conditions for partial differential equations with delay to generate $C_{0}$-semigroups // J. Differential Equations. 1983. V. 50. № 1. P. 49-79.

[2] Desch W., Schappacher W. Spectral properties of finite-dimensional perturbed linear semigroups // J. Differential Equations. 1985. V. 59. №1. P. 80-102.

[3] Власов В.В. Об одном классе дифференциально-разностных уравнений в гильбертовых пространствах и некоторых спектральных вопросах // Докл. РАН. 1992. Т. 327. № 4-6. C. $428-432$.

[4] Власов В.В. Корректная разрешимость одного класса дифференциальных уравнений с отклоняющимся аргументом // Изв. ВУЗов. Сер. матем. 1996. № 1. С. 22-44.

[5] Скубачевский А. Л. О некоторых свойствах эллиптических и параболических функционально-дифференциальных уравнений // УМН. 1996. Т. 51. №1. С. 169-170.

[6] Skubachevskii A. L. Bifurcation of periodic solutions for nonlinear parabolic functional differential equations arising in optoelectronics // Nonlinear Anal. 1998. V. 32. № 2. P. 261-278.

[7] Скубачевский А. Л., Шамин Р. В. Первая смешанная задача для параболического дифференциально-разностного уравнения // Матем. заметки. 1999. Т. 66. № 1. С. 145-153.

[8] Борок В. М., ЖКитомирский Я.И. О задаче Коши для линейных уравнений в частных производных с линейно преобразованным аргументом // Докл. АН СССР. 1971. Т. 200. № 3. C. $515-518$.

[9] Муравник А. Б. О задаче Коши для некоторых дифференциально-разностных уравнений параболического типа // Докл. РАН. 2002. Т. 385. № 5. С. 604-607.

[10] Razgulin A. V. Rotational multi-petal waves in optical system with 2-D feedback // Chaos in Optics. Proceedings SPIE. 1993. V. 2039. P. 342-352.

[11] Vorontsov M. A., Iroshnikov N. G., Abernathy R. L. Diffractive patterns in a nonlinear optical two-dimensional feedback system with field rotation // Chaos, Solitons, and Fractals. 1994. V. 4. P. 1701-1716. 